\title{
TEMA 4-2014: Anestesia y Cirugía Bariátrica: Más Que Obesidad
}

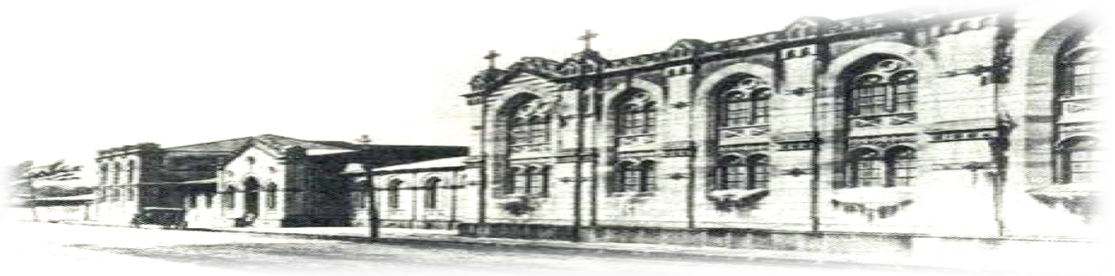

Haspital San quan de Dias. San Jasé. Casta Rica. Fundada en 1845

Recibido: $\quad$ 05/02/2014

Aceptado: $\quad 26 / 03 / 2014$

Diana Morales ${ }^{1}$

${ }^{1}$ Médica Residente del Posgrado de Anestesiología y Recuperación. Hospital México. UCR-CENDEISSSCCSS. Correo electrónico: dmc2307@hotmail.com

\section{RESUMEN}

Existe una epidemia de sobrepeso que afecta aproximadamente a 1.7 billones de personas alrededor del mundo, de ellos el 50\% son obesos. La obesidad es una patología asociada a gran cantidad de comorbilidades, su aumento ha llevado al desarrollo de múltiples procedimientos quirúrgicos para el control de la misma. Cada pacientes sometido a cirugía bariátrica es un reto para el anestesiólogo ya que presenta un riesgo aumentado de complicaciones, que va mas allá del aumento del peso per se.

\section{PALABRAS CLAVE}

Obesidad. Apnea obstructiva del sueño. Cirugía bariátrica.

\begin{abstract}
There's a worldwide overweight outbreak that affects approximately 1, 7 billion of people, $50 \%$ of them are obese. The obesity is associated to numerous comorbidities; this increase has lead to the development of multiple surgical procedures for the control of the disease. Each patient that undergo bariatric surgery is a challenge for the anesthesiologist because he poses an increased risk for major complications beyond the weight.
\end{abstract}

\section{KEY WORDS}

Obesity. Obstructive sleep apnea. Bariatric surgery. 


\section{INTRODUCCIÓN}

Existe una epidemia de sobrepeso que afecta aproximadamente a 1.7 billones de personas alrededor del mundo, actualmente el número de personas con sobrepeso es igual al número de personas con desnutrición. La obesidad ya no significa opulencia sino un problema de salud pública. En Estados Unidos, dos tercios de la población posee sobrepeso y de ellos al menos el $50 \%$ son obesos, esto representa más de 50 millones de estadounidenses, en Inglaterra entre 1993 y el 2004 la obesidad aumentó de un 13.6 a un $24 \%$ en hombres y de un 16 a un $24.4 \%$ en mujeres $^{(1,2)}$.

El aumento de la incidencia de esta patología ha llevado al reconocimiento de múltiples síndromes relacionados a la misma. La cirugía bariátrica se ha vuelto el pan de cada día de los cirujanos, por ejemplo en 1990 se realizaron 16.000 procedimientos en Estados Unidos, para el 2008 habían ascendido a más de 220.000, el $90 \%$ de ellos mínimamente invasivos. El paciente obeso presenta retos particulares para el anestesiólogo y, conforme las intervenciones bariátricas aumentan, éste debe enfrentarse a esta población $^{(3,4)}$.

\section{DISCUSIÓN}

\section{Generalidades}

La obesidad es una enfermedad crónica y multifactorial que deriva de la interacción entre el genotipo y el medio ambiente. En 1997 la Organización Mundial de la Salud la definió como un exceso de acumulación de grasa que puede afectar negativamente la salud. La mayoría de los sistemas y órganos del cuerpo se ven afectados por esta patología. El sistema cardiovascular por hipertensión arterial, enfermedad aterosclerótica, enfermedad vascular cerebral, insuficiencia venosa periférica, tromboflebitis y embolismo pulmonar; el sistema respiratorio por asma, apnea obstructiva del sueño y síndrome de hipoventilación de la obesidad; a nivel metabólico se observan diabetes tipo 2, intolerancia a los carbohidratos e hiperlipidemia; a nivel musculoesquelético herrnias de disco y osteoartritis de caderas y rodillas; a nivel gastrointestinal colelitiasis, reflujo gastroesofágico, hepatitis no alcohólica, carcinoma hepático y carcinoma colorectal; a nivel urológico incontinencia urinaria; en el sistema reproductivo ovario poliquístico, infertilidad, anormalidades fetales, hipogonadismo y cáncer de endometrio, ovario y próstata $\mathrm{y}$ a nivel psicológico depresión $\mathrm{y}$ trastornos de la alimentación ${ }^{(1,4,5)}$.

El sobrepeso se define como un índice de masa corporal $\left(\right.$ IMC) $>25 \mathrm{y}<30 \mathrm{~kg} / \mathrm{m}^{2}$, la obesidad se define como un IMC $>30 \mathrm{~kg} / \mathrm{m}^{2}$ y se clasifica como grado I (IMC de 30-34.9 Kg/m²), grado II (IMC $35-39.9 \mathrm{Kg} / \mathrm{m}^{2}$ ) y grado III $\mathrm{u}$ obesidad mórbida (IMC $\left.\geq 40 \mathrm{~kg} / \mathrm{m}^{2}\right)^{(3)}$.

La súper obesidad se define como un $\mathrm{IMC}>50$ $\mathrm{kg} / \mathrm{m}^{2}$ y la ultra obesidad como IMC $>70$ $\mathrm{kg} / \mathrm{m}^{(2,6)}$. En los niños, el sobrepeso se define como un IMC > el percetilo 95 para la edad. Entre 1970 y 2000 el sobrepeso en niños se triplicó, actualmente aproximadamente el 15\% de los niños entre los 6 y 9 años de edad lo $\operatorname{padecen}^{(1)}$.

La distribución del tejido adiposo parece tener mayor relevancia clínica que el peso o el índice de masa corporal, la obesidad central o androide es metabólicamente más activa que la periférica, existe una mayor tasa de complicaciones en hombres con una circunferencia abdomial $>102$ $\mathrm{cm}$ y mujeres con una circunferencia abdomial $>$ $89 \mathrm{~cm}^{(2)}$.

El síndrome metabólico se caracteriza por obesidad truncal asociada a resistencia a la insulina, control lipídico alterado, disminución de los niveles de HDL y aumento de los triglicéridos, y/o hipertensión arterial. La patogénesis involucra el acúmulo de tejido adiposo que secreta adipocinas proinflamatorias, aumento de los niveles de interleucina 6 y factor de necrosis tumoral $\alpha$. La hiperglicemia es el signo asociado a mayor mortalidad ${ }^{(7)}$.

El estudio de Framingham reveló que por cada onza de peso que se gana entre los 30 y 42 años de edad, aumenta un $1 \%$ la mortalidad. En Estados Unidos solo uno de cada siete personas obesas alcanzara la expectativa de vida. En los obesos mórbidos la expectativa de vida es 9 años menor en las mujeres y 12 años en los hombres ${ }^{(1)}$.

Los costos de salud asociados a la obesidad ascienden a más de 140 billones de dólares anualmente en Estados Unidos. Los estudios han demostrado que la terapia médica logra una 
reducción máxima del $10 \%$ del peso corporal en un 10 a $40 \%$ de los pacientes. Pérdidas moderadas de tan solo el 5\% del peso conllevan beneficios considerables en la salud, sin embargo son difíciles de mantener a lo largo del tiempo. La cirugía bariátrica se refiere a la variedad de procedimientos quirúrgicos empleados para lograr la pérdida de peso y es la terapia más efectiva contra la obesidad ${ }^{(1,5)}$.

\section{Cirugía Bariátrica}

Según el concenso de la conferencia sobre cirugía bariátrica llevada a cabo en 2004, ésta es la terapia más efectiva para tratar la obesidad mórbida y puede llevar a la mejoría e incluso cura completa de las comorbilidades de la obesidad $^{(1)}$. Los candidatos a la cirugía deben haber sido instados a perder peso por medios no quirúrgicos como dieta, atención nutricional y programas hospitalarios de pérdida de peso por al menos seis meses. La cirugía debe ser ofrecida a pacientes con obesidad mórbida o pacientes con obesidad grado II que asocien comorbilidades que pueden resolver gracias a la pérdida de peso, por ejemplo diabetes mellitus, apnea obstructiva del sueño y enfermedad arterial coronaria. La cirugía se recomienda como primera línea de tratamiento en obesos súper mórbidos $\left(\mathrm{IMC} \geq 50 \mathrm{~kg} / \mathrm{m}^{2}\right)^{(1,2,5)}$.

Actualmente existen cuatro tipos de procedimientos utilizados para la pérdida sostenida de peso: el bypass gástrico, solo o en combinación con la gastroplastía vertical; la banda gástrica ajustable y la derivación biliopancreática con "cruce" duodenal ${ }^{(1)}$. Las contraindicaciones para la cirugía incluyen impedimento mental como depresión mayor o psicosis, abuso de sustancias, enfermedad hepática avanzada con hipertensión portal, malignidad con pobre pronóstico a 5 años, enfermedad arterial coronaria inestable y OSA con hipertensión pulmonar ${ }^{(2,5)}$.

Los procedimientos pueden realizarse laparoscópicamente o abiertos, la laparotomía posee ciertas ventajas como el control táctil de la disección, la habilidad de palpar los tejidos, libertad para la utilización de diversas suturas, técnicas y materiales y menor tasa de compliaciones como hemorragias, fugas anastomóticas, hernias y obstrucción intestinal. El procedimiento laparoscópico reduce el tiempo de estancia hospitalaria, hay menos cicatrices y dolor, presenta menos tasa de infecciones de herida quirúrgica, hernia incisional y dehiscencia de herida, sin embargo, aumenta las complicaciones intraabdominales ${ }^{(1,5)}$.

El SOS (Swedish Obese Subjects Intervention Study) fue un estudio que como su nombre indica se realizó en Suecia y comparó pacientes que fueron tratados con cirugía bariátrica $\mathrm{y}$ tratamiento convencional. Al analizar los resultados, el grupo quirúrgico presentó menores tasas de hipertensión, diabetes mellitus, e hipertrigliceridemia a los dos años ${ }^{(8)}$. Cuando se analizaron los datos a 10 años plazo, se observó una reducción del $16 \%$ del peso corporal, la escala de salud mejoró en un $11 \%$ de los pacientes y los problemas psciológicos asociados a la obesidad mejoraron en un $49 \%$ de ellos, demostrando los beneficios a largo plazo de la cirugía bariátrica $^{(8,9)}$.

Laurino y colaboradores dieron seguimiento a 379 pacientes sometidos a bypass gástrico $\mathrm{y}$ demostraron una mejoría que se mantuvo a lo largo de 5 años en la incidencia de diabetes (reducción de un 14\%), hipertensión (reducción de un 35\%), enfermedad cardiovascular (reducción de un 5\%) e infertilidad (reducción de un $4 \%$ ), esta mejoría se relacionó al porcentaje de peso perdido. Los autores encontraron un tasa de falla terapéutica entre 7 a $20 \%{ }^{(10)}$.

El bypass gástrico es el procedimiento más utilizado en el mundo, representa aproximadamente el $80 \%$ de las cirugías bariátricas, fue introducido por el Dr. Edward Mason en 1969 y realizado laparoscópicamente en 1990 por el Dr. Alan Wittgrove. El procedimiento consiste en una reducción del volumen gástrico que conlleva a una restricción de la ingesta de comida, disminuyen también los niveles de grelina y aumentan los del péptido $\mathrm{Y}^{(6)}$.

La pérdida de peso después de este procedimiento ronda el 65 a $70 \%$ del exceso de peso corporal, alrededor de un $35 \%$ del IMC. La pérdida de peso usualmente alcanza el nadir en 1 a 2 años. La mortalidad perioperatoria ronda el $0.5 \%$ y la morbilidad el $5 \%$. Las complicaciones respiratorias y cardíacas rondan el 4 a $7 \%$ y el 1 a $4 \%$ respectivamente, la tasa de reintervención varía entre un 6 y un $9 \%{ }^{(1,2)}$.

La banda gástrica ajustable es el procedimiento menos invasivo y el utilizado mayormente en 
Europa, Australia y Suramérica. Consiste en la colocación de una banda elástica en el estómago y un mango inflable a nivel de la fascia abdominal donde se inyecta solución salina para restringir el volumen gástrico ${ }^{(5)}$. O'Briens y colaboradores demostraron una reducción del exceso de peso corporal de un $51 \%$ a los 12 meses y de un $68 \%$ a los 40 meses. El procedimiento conlleva una mortalidad de un $0.1 \%$ y una mortalidad de $5 \%{ }^{(1,5)}$.

La gastroplastía vertical se introdujo en 1970, es relativamente sencilla y rápida, lleva a una pérdida del 50 a $60 \%$ del exceso de peso corporal y un 25 a $30 \%$ del IMC. Presenta una mortalidad de $0.1 \%$ y una morbilidad de $5 \%{ }^{(1)}$.

La derivación biliopancreática es un procedimiento principalmente malabsortivo, es una cirugía compleja por lo que requiere cirujanos experimentados, consiste en una gastrectomía distal y la formación de una gastroileostomía. Se utiliza en pacientes extremadamente obesos con IMC $\geq 60 \mathrm{~kg} / \mathrm{m}^{2}$. Hess y colaboradores demostraron una pérdida del $70 \%$ del exceso de peso corporal, incluso 8 años después del procedimiento, en 440 pacientes. La cirugía asocia una mortalidad de $1 \%$ y morbilidad de $9 \%{ }^{(5)}$.

\section{Manejo Preoperatorio}

Como la obesidad por sí misma es difícil de tratar, la disminución del riesgo perioperatorio puede ser difícil. La finalidad de la valoración preoperatoria es optimizar los resultados del paciente y el tratamiento de las condiciones médicas. No existe evidencia de que la visita preanestésica un día previo a la cirugía tenga beneficios en los resultados del paciente, sin embargo posibles ventajas son mayor satisfacción del usuario, identificación temprana de posibles dificultades en el manejo de la vía aérea y obtención de accesos vasculares, detección de AOS y disminución en la cancelación de los casos. La visita preanestésica se recomienda de 6 a 12 semanas previo a la cirguía $^{(2,7)}$.

La obesidad por sí sola no siempre aumenta la morbimortalidad perioperatoria, no obstante los pacientes con síndrome metabólico sometidos a cirugía bariátrica presentan 2.5 veces mayor mortalidad, de 3 a 7 veces más casos de insuficiencia renal aguda y 4 veces más eventos cerebrovasculares $^{(3)}$.

Los exámenes de laboratorio recomendados son hematocrito, glucosa y pruebas de función renal, hepática y tiroidea con un máximo 6 meses de vigencia, otros análisis serán necesarios dependiendo del ASA del paciente ${ }^{(3,7)}$.

El peso y el IMC son pobres predictores de vía aérea difícil, la circunferencia del cuello $>40 \mathrm{~cm}$, un Mallampati $\geq 3$ y una distancia tiromentoniana $<6 \mathrm{~cm}$ son los indicadores más específicos, para predecir posibles dificultades durante la intubación. Miller sugiere que en un $25 \%$ de los reclamos por vía aérea difícil en pacientes obesos, no hubo valoración preanestésica $^{(2)}$.

Los pacientes obesos poseen un consumo basal de oxígeno y una producción de $\mathrm{CO}_{2}$ aumentados, también asocian disminución de la compliance pulmonar, aumento de la resistencia en la vía aérea y disminución de la capacidad funcional residual $^{(2,11)}$. La menor capacidad de cierre resulta en atelectasias. La posición supina y el neumoperitoneo acentúan estas alteraciones ${ }^{(2)}$. Las pruebas de función pulmonar rara vez modifican el manejo anestésico puesto que la mayoría de los pacientes van a tener enfermedad pulmonar restrictiva. La utilidad de la radioigrafía de tórax y los gases arteriales es poca, estos últimos suelen mostrar la hipoxia y la hipercapnia crónicas ${ }^{(7)}$.

El cese del fumado preoperatorio puede disminuir el riesgo operatorio, mejorar los parámetros cardiovasculares y disminuir las complicaciones posoperatorias, se recomienda la suspensión seis a ocho semanas antes de la cirugía $^{(2-4)}$.

Más del 70\% de los pacientes que se someten a cirugía bariátrica padecen de apnea obstrictuva del sueño (AOS), este es un síndrome caracterizado, como su nombre bien lo inidica, por obstrucción de la vía aérea superior durante el sueño ${ }^{(6)}$. El padecimiento debe pensarse según la historia clínica, presencia de apneas presenciadas, ronquidos, trastornos del sueño y cansancio crónico, se recomienda realizar los cuestionarios STOP y la escala de Epworth, y referir a los pacientes con alta sospecha para que se les realice una polisomnografía. La polisomnografía resulta positiva por AOS si se 
presenta más de 5 apneas de 10 segundos por hora $^{(2,3)}$.

La hipoxemia crónica resultante de la apnea del sueño lleva a aumento del flujo sanguíneo pulmonar, policitemia e hipertensión pulmonar, lo que deriva en hipertrofia ventricular derecha, asimismo existe un riesgo aumentado para $\operatorname{arritmias~cardíacas~}^{(6,11)}$.

El tratamiento crónico de la AOS consiste en presión positiva de la vía aérea (CPAP) o presión positiva binivel (BiPAP) durante 6 a 12 semanas. Los estudios han demostrado que en 2 semanas de CPAP mejora la actividad respiratoria, en 4 semanas mejora el control de la presión arterial y la frecuencia cardíaca, en 6 semanas aumenta la cavidad faríngea y disminuye el volumen de la lengua, en 8 semanas disminuye el riesgo cardiovascular y mejora la hipertensión $\operatorname{pulmonar}^{(4,7)}$.

La evaluación del sistema cardiovascular puede ser complicada, los sonidos cardiacos se encuentran abolidos, la ingurgitación yugular es difícil de observar y cuesta diferenciar el edema periférico del tejido adiposo. Es mejor establecer la capacidad funcional del pacientes según su habilidad para llevar a cabo actividades de la vida diaria, aquellos con una capacidad $>4$ METs (equivalentes metabólicos), por ejemplo subir escaleras o una cuesta son clasificados con una capacidad funcional moderada. El índice cardíaco revisado de Lee se utiliza comúnmente para establecer el riesgo de los pacientes. Los pacientes con capacidad funcional moderada y bajo riesgo en la escala de Lee poseen bajo riesgo de complicaciones cardiovasculares. En pacientes con capacidad restringida para las actividades diarias pueden realizarse tests farmacológicos como el ecocardiograma de estrés con dobutamina. La cateterización cardíaca se considera el patrón de oro para detectar enfermedad arterial coronaria ${ }^{(2)}$.

Existen otras consideraciones que se deben tomar en cuenta. El control glicémico es recomendado para reducir los eventos adversos. Las neuropatías preexistentes como el síndrome del túnel carpal deben ser documentadas ${ }^{(2)}$.

La escala de riesgo de mortalidad para la cirugía de obesidad (OS-MRS por sus siglas en inglés) fue validada recientemente para calcular la mortalidad perioperatoria. Esta incluye cinco variables: IMC > $50 \mathrm{~kg} / \mathrm{m}^{2}$, género masculino, hipertensión arterial sistémica, factores de riesgo para embolismo pulmonar y edad $\geq 45$ años. Un paciente con 0-1 puntos es considerado de bajo riesgo o clase $\mathrm{A}$, con una mortalidad de $0.31 \%$; un paciente con 2 a 3 puntos se encuentran en riesgo intermedio, clase $\mathrm{B}, \mathrm{y}$ posee una mortalidad de $1.9 \%$ y los pacientes con 4 a 5 puntos son la clase de alto riesgo o $\mathrm{C}$ y presentan una mortalidad de $7.5 \%$, lo que representa una mortalidad 12 veces mayor que el grupo de bajo riesgo $^{(2)}$.

\section{Manejo Transoperatorio}

La ventilación con mascarilla facial y la intubación suelen ser complicadas en el paciente obeso. La inducción de la anestesia tiene el potencial de ser particularmente difícil debido a un aumento de la incidencia de vía aérea difícil y riesgo de aspiración del contenido gástrico ${ }^{(6)}$. Juvin y colaboradores han demostrado mayor incidencia de intubación difícil en pacientes obesos así como mayor riesgo de desaturaciones ${ }^{(3)}$. Brosky reporta que un paciente con una circunferencia del cuello $>40 \mathrm{~cm}$ tiene una probabilidad de $5 \%$ de intubación difícil, esta probabilidad asciende a un $35 \%$ en un paciente con una circunferencia $>60 \mathrm{~cm}^{(12)}$. La obesidad severa se asocia a intubación prolongada, dificultad para el destete del ventilador y mayor estancia en la unidad de cuidados intensivos así como hospitalaria ${ }^{(2)}$.

Se recomienda preoxigenar a los pacientes por tres minutos en posición de trendelemburg inverso a $30^{\circ}$, la posición se ha asociado con menor riesgo de aspiración de contenido gástrico al disminuir la presión intraabdominal ${ }^{(12)}$. La presión positiva al final de la espiración (PEEP) de $10 \mathrm{cmH}_{2} \mathrm{O}$ por 5 minutos o la presión positiva no invasiva previas a la intubación han demostrado prolongar la duración de la apnea no hipóxica $^{(2,4)}$. Los períodos de hipoxemia e hipercapnia pueden aumentar las resistencias vasculares pulmonares y precipitar insuficiencia cardíaca derecha. Estrategias como las maniobras de reclutamiento, presión positiva al final de la espiración, posición fowler y CPAP postoperatorio ayudan a prevenir las complicaciones pulmonares ${ }^{(3)}$.

Los pacientes obesos son más propensos a sufrir atelectasias y desaturaciones debido a la pobre compliance pulmonar ${ }^{(7)}$. Los volúmenes tidales 
excesivos (mayores a $20 \mathrm{ml} / \mathrm{kg}$ ) resultan en hipocapnia excesiva y aumentan las presiones de la vía aérea sin mayores beneficios en la oxigenación. Se recomienda entonces volúmenes de $10 \mathrm{ml} / \mathrm{kg}$ según el peso ideal del paciente para minimizar el volutrauma, en conjunto con PEEP moderado. Cualquier acidosis respiratoria puede activar el sistema simpático causando taquicardia, disritmias y aumento de las resistencias vasculares sistémicas y pulmonares ${ }^{(2)}$.

En estos pacientes se recomienda secuencia rápida de inducción, disponibilidad de dispositivos de ventilación supraglóticos como la mascarilla laríngea Fast-Trach o proseal y personal capacitado para ayudar en caso de una vía aérea difícil. El uso de propofol y un relajante neuromuscular como el rocuronio basados en el peso ideal se recomiendan para la inducción. Se ha demostrado que los pacientes obesos poseen volúmenes gástricos residuales mayores a $25 \mathrm{ml}$ y $\mathrm{pH}$ menores a 2.5 lo que los coloca en riesgo de bronconeumonías por aspiración, algunos autores recomiendan utilización de presión en el cricoides para la intubación y la colocación de una sonda naso $\mathrm{u}$ orogástrica previa a la manipulación quirúrgica ${ }^{(2,3)}$.

El volumen de distribución para las drogas lipofílicas como los barbitúricos y benzodiacepinas se encuentra aumentado. La unión a la albúmina y la fracción libre de los fármacos parecen no verse alterados. La vida media de eliminación del propofol es similar en pacientes no obesos y obesos, se recomienda calcular la dosis según el peso actual del paciente $^{(11)}$.

Se ha demostrado que la dosificación del fentanil según el peso actual del paciente sobreestima los requerimientos del mismo. Dos estudios aleatorizados, randomizados y controlados demostraron bloqueo neuromuscular prolongado debido al uso de cisatracurio y rocuronio según peso actual. Por estos motivos se recomienda la dosificación de los medicamentos según el índice de masa magra (aproximadamente el 120\% del peso ideal $)^{(3,11)}$.

Los gases anestésicos sevoflurano, desflurano e isoflurano pueden ser utilizados con seguridad en estos pacientes $^{(12)}$. La baja solubilidad en sangre del desflurano facilita el emerger de la anestesia y que se restablezcan los reflejos de la vía aérea. No se ha encontrado diferencias en pacientes obesos mórbidos mantenidos bajo anestesia con sevoflurano, desflurano o infusión de propofol $^{(4,6)}$.

Los a2-agonistas como la clonidina y la dexmedetomidina disminuyen los requerimientos anestésicos y analgésicos intra y posoperatorios sin causar depresión respiratoria por lo que pueden utilizarse como coadyuvantes, la hipotensión puede ser un efecto adverso ${ }^{(4)}$.

El uso de óxido nitroso está limitado en la cirugía bariátrica debido al riesgo de distención abdomial y por el neumoperitoneo. El neumopertitoneo creado por los procedimientos laparoscópicos mayor a $20 \mathrm{mmHg}$ causa compresión de la vena cava inferior y disminución del retorno venoso y el gasto cardíaco $^{(2)}$.

El régimen ideal de fluidos se desconoce, ni el restrictivo $(4 \mathrm{ml} / \mathrm{Kg} / \mathrm{h})$ ni el liberal $(12 \mathrm{ml} / \mathrm{kg} / \mathrm{h})$ han mostrado superioridad. El gasto urinario usualmente se encuentra disminuido en los procedimientos laparoscópicos ${ }^{(2)}$.

El calentamiento térmico y la euvolemia han demostrado mantener la homeostasis y adecuada perfusión de los órganos, la hipotermia se ha asociado a mayor tasa de infecciones de herida quirúrgica, coagulopatía, despertar prolongado y morbilidad cardiaca ${ }^{(2)}$.

La monitorización de la presión arterial puede ser difícil debido al gran tamaño y forma cónica de los brazos, puede incluso ser necesaria la medición de presión arterial invasiva. La ultrasonografía debe ser utilizada para la canulación de vías centrales en cuello. La rabdomiolisis se ha reportado desde un 12 hasta un $37 \%$ y se asocia con tiempos quirúrgicos prolongados (mayores a 240 minutos), IMC $>50$ $\mathrm{kg} / \mathrm{m}^{2}$ y cirugía abierta ${ }^{(2,3)}$.

\section{Cuidado Postanestésico}

Las consideraciones postoperatorias incluyen soporte ventilatorio y de la vía aérea, manejo del dolor y prevención del tromboembolismo ${ }^{(2)}$.

Los pacientes con apnea obstructiva del sueño (AOS) son más propensos a obstrucción de la vía aérea y depresión respiratoria. El manejo de la vía aérea, la analgesia y las decisiones para el alta hospitalaria son más difíciles. Los pacientes 
son más susceptibles a los sedantes, hipnóticos y opioides, probablemente debido a los cambios en los niveles cerebrales de adenosina ${ }^{(2,3)}$.

La extubación conlleva serios riegos de pérdida del control de la vía aérea, rápida aparición de hipoxemia, inestabilidad hemodinámica y broncoaspiración ${ }^{(2)}$. Para el despertar se sugiere el paciente se encuentre completamente despierto y el bloqueo neuromuscular adecuadamente revertido ${ }^{(3)}$. Huerta y colaboradores han reportado el uso de CPAP postoperatorio luego de un bypass gástrico sin aumentar el riesgo de fuga de las anastomosis. El CPAP y el BiPAP deben ser utilizados según sea necesario, estos reducen el riesgo de reintubación, la estancia en la unidad de cuidado intensivo, neumonías, sepsis e infección ${ }^{(4)}$.

La analgesia va a depender de si la cirugía fue abierta o laparoscópica. No se recomienda el uso de opioides de acción prolongada, sobre todo en pacientes con AOS o síndrome de hipoventilación pulmonar. La anestesia epidural cuando se ha realizado laparotomía disminuye las complicaciones pulmonares y el requerimiento de opioides. La anestesia regional con anestésicos locales se asocia a menores requerimientos de opioides ${ }^{(7,13)}$. El bloqueo del transverso abdomial alto guíado por ultrasonografía ha demostrado ser una herramienta útil para la analgesia ${ }^{(2)}$. La utilización de antiinflamatorios no esteroideos como el ketorolaco disminuye los requerimientos de opioides. La analgesia controlada por el paciente es eficaz y segura ${ }^{(3)}$. Otros medicamentos utilizados son la ketamina, clonidina, dexmedetomidina, sulfato de magnesio, metilprednisolona y lidocaína ${ }^{(4)}$.

Los pacientes obesos tienen un riesgo cuatro veces mayor de trombosis venosa profunda. El antecedente de TVP o signos clínicos de la misma sugieren la necesidad de realizar los análisis correspondientes. $\mathrm{Si}$ una TVP es diagnosticada la cirugía debe retrasarse un mes y el paciente debe ser anticoagulado ${ }^{(7)}$.

Posterior a la cirugía las medias de compresión neumática, la movilización temprana, altas dosis de heparina, aspirina o heparinas de bajo peso molecular son las medidas de profilaxis necesarias para la $\operatorname{TVP}^{(2,14)}$. La dosificación de la heparina según el peso actual del paciente puede sobreestimar las necesidades, sin embargo las dosis fijas suelen ser insuficientes para algunos pacientes. Scholtem y colaboradores demostraron que $40 \mathrm{mg}$ subcutáneos de enoxaparina dos veces al día son efectivos para prevenir trombosis venosa profunda sin aumentar el riesgo de sangrado, sin embargo Hamada recomienda calcular la dosis de enoxaparina a $1.5 \mathrm{mg} / \mathrm{kg}$ una vez al día o 1 $\mathrm{mg} / \mathrm{kg}$ dos veces al día, la terapia debe continuarse 2 a 4 semanas $^{(14,15)}$.

A los pacientes con alto riesgo de tromboembolismo pulmonar debe colocárseles un filtro de vena cava inferior, éstos son aquellos con IMC > $60 \mathrm{~kg} / \mathrm{m}^{2}$, historia de AOS o tromboembolismo previo $^{(2)}$. Si un paciente inicia con hipotensión, disnea y dolor torácico debe pensarse en TEP. El dímero $\mathrm{D}$ no es útil en pacientes de alto riesgo hospitalizados, la examinación ecocardiográfica puede mostrar el émbolo a nivel de la arteria pulmonar y/o disfunción ventricular derecha ${ }^{(7)}$.

\section{CONCLUSIONES Y RECOMENDACIONES}

La obesidad es una patología cuya prevalencia va en aumento, debido a la escaza eficacia del tratamiento médico se ha dado un auge de la cirugía bariátrica.

El aumento en el número de estos procedimientos que se realizan conlleva a una necesidad de que los anestesiólogos sean más versados en el manejo de los pacientes obesos y sus complicaciones perioperatorias.

\section{REFERENCIAS BIBLIOGRÁFICAS}

1. Buchwald H. Consensus Conference Statement Bariatric surgery for morbid obesity: Health implications for patients, health professionals, and thirdparty payers. Surgery for Obesity and Related Diseases 2005;1:371-381.

2. O'neill T Allam J. Anesthetic considerations and management of the obese patient presenting for bariatric surgery. Current Anesthesia \& Critical Care 2010;21:16-23.

3. Schumman R. Anesthesia for bariatric surgery. Best Practice \& Research Clinical Anesthesiology. 2011; 25:83-93.

4. Schumman R Jones R Ortiz V et al. Best Practice Recommendations for An- 
esthetic Perioperative Care and Pain Management in Weight Loss Surgery. Obesity Research 2005;13:254-266.

5. Khwaja H Bonanomi G. Bariatric surgery: techniques, outcomes and complications. Current Anesthesia \& Critical Care. 2010;21:31-38.

6. Candiotti K Sharma S Shankar R. Obesity, obstructive sleep apnea and diabetes mellitus, anesthetic implications. British Journal Anesthesia 2009;103: 23-30.

7. Tung A. Anesthetic Considerations with the Metabolic Syndrome. British Journal of Anesthesia 2010;105:i24-i33.

8. Sjostrom D Lissner L Sjostrom L. Reduction in Incidence of Diabetes, Hypertension and Lipid disturbances after Intentional Weight Loss Induced by Bariatric Surgery: the SOS Intervention Study. Obesity Research 1999;7:477484.

9. Karlsson J Taft C Rydén A Sjostrom L Sullivan M. Ten-year trends in Healthrelated quality of life after surgical and conventional treatment for severe obesity: the SOS intervention study. International Journal of Obesity 2007;31:12481261.

10. Laurino R Herbella F Tauil R Silva F Lima S. Comorbidities Remission After Roux-en-Y Gastric Bypass for Morbid Obesity is Sustained in a Long-Term Follow-up and correlates with Weight Regain. Obesity Surgery 2012;22:15801585.

11. Passannante A Tielborg, M. (2009). Anesthetic Management of Patients with Obesity with and without Sleep Apnea. Clinical Chest Medicine 2009;30:569579.

12. Brodsky J Lemmens H Brock-Utne J Vierra M Saidman J. (2002). Morbid Obesity and Tracheal Intubation. Anesthesia \& Analgesia 2002;94:732-736.

13. Ingrande $\mathrm{J}$ Brodsky $\mathrm{J}$ Hendrikus. Regional Anesthesia and Obesity. Current Opinion Anesthesiology 2009;22:683686.

14. Hamada G Smith P. Eoxaparin for Thromboprophylaxis in Morbidly Obese Patients Undergoing Bariatric Surgery: Findings of the Prophylaxis Against VTE Outcomes in Bariatric Surgery Pa- tients Receiving Enoxaparin Study. Obesity Surgery 2005;15:1368-2374.

15. Sheperd M Rosborough T Schwartz M. Heparin Tromboprophylaxis in Gastric Bypass Surgery. Obesity Surgery 2003;13:249-253.

\section{DECLARACIÓN DE CONFLICTO DE IN- TERESES}

La autora declara que no existe conflictos de interés. 